\title{
Politique
}

Politique

\section{Caroline ANDREW et Beth MILROY (Eds), Life Spaces, Gender, Hoursehold, Employment, Vancouver, University of British Columbia Press, 1988, 214 p.}

\section{Annick Germain}

Numéro 17, hiver 1990

Les nouveaux enjeux du politique

URI : https://id.erudit.org/iderudit/040656ar

DOI : https://doi.org/10.7202/040656ar

Aller au sommaire du numéro

Éditeur(s)

Société québécoise de science politique

ISSN

0711-608X (imprimé)

1918-6584 (numérique)

Découvrir la revue

Citer ce compte rendu

Germain, A. (1990). Compte rendu de [Caroline ANDREW et Beth MILROY (Eds), Life Spaces, Gender, Hoursehold, Employment, Vancouver, University of British Columbia Press, 1988, 214 p.] Politique, (17), 167-171.

https://doi.org/10.7202/040656ar d'utilisation que vous pouvez consulter en ligne.

https://apropos.erudit.org/fr/usagers/politique-dutilisation/ 
Caroline ANDREW et Beth MILROY (Eds), Life Spaces, Gender, Hoursehold, Employment, Vancouver, University of British Columbia Press, 1988, 214 p.

L'analyse féministe des années 80 serait-elle, dans le champ des études urbaines, ce que fut l'analyse des classes sociales dans les années 70, à savoir une problématique radicalement nouvelle, porteuse à la fois de regards inédits sur la ville et de politiques renouvelées face aux grandes questions urbaines? Ou vient-elle seulement s'ajouter aux sous-champs de recherche qui se sont accumulés depuis bientôt vingt ans dans le domaine des études urbaines et régionales, ou encore nous en propose-t-elle une relecture à la faveur d'une position analytique privilégiée fondée sur l'irréductibilité des différences qui séparent l'expérience qu'ont les femmes et les hommes de la ville et de ses problèmes?

En centrant leur livre sur «les interrelations entre le genre (gender) et les structures urbaines», et en conviant dix chercheurs canadiens à y présenter leurs travaux, Caroline Andrew et Beth Milroy ont posé un geste fondateur important puisque Life Spaces est le premier véritable ouvrage collectif publié au Canada sur l'approche féministe canadienne en études urbaines. En 1975, la Commission de la capitale nationale avait déjà organisé un atelier national sur les femmes et l'environnement urbain et le ministère d'État aux Affaires urbaines avait, à l'époque, financé quelques études sur le sujet. (Ces initiatives n'étaient probablement pas sans rapports avec l'Année internationale de la femme!). Il faudra pourtant attendre les années 80 pour assister à l'essor de recherches sur l'impact des structures urbaines sur les conditions de vie des femmes (et réciproquement). L'ouvrage d'Andrew et Milroy présente un excellent échantillon de ces recherches mais soulève aussi un certain nombre de questions quant aux contours et aux méthodes de ce champ d'études.

Le premier texte, écrit par Suzanne Mackenzie, nous plonge dans le vif du sujet puisqu'il aborde l'évolution des liens entre les formes urbaines, l'articulation des sphères de la production et de la reproduction, et la question des femmes, telle qu'elle s'est cristallisée autour de deux périodes de crise: le tournant du siècle et 
la période actuelle. Propos ambitieux appliqué à un survol historique plutôt large, ce texte présente une problématique de l'urbanisation centrée sur le rôle des femmes. La restructuration de l'économie autour de l'industrialisation de la fin du siècle dernier provoque l'entrée des femmes sur le marché du travail salarié et de façon plus générale une redéfinition substantielle de la vie quotidienne des ménages désormais scindée en sphères distinctes. Cette séparation de la vie domestique et de la vie de travail engendre pour les familles toute une série de problèmes qui se traduisent bientôt en «questions sociales» dont la question féminine. Pour résoudre, du moins en partie, les dilemmes dans lesquels sont plongées les familles, une réorganisation du paysage urbain s'impose et c'est l'avènement des banlieues, assorties de toute une panoplie d'infrastructures nouvelles au service de la famille et des arts ménagers. Mais cette solution est onéreuse et les femmes devront réintégrer le marché du travail à la fois pour permettre au ménage de joindre les deux bouts et combler les besoins en maind'oeuvre suscités par cette nouvelle économie de consommation. Un grand nombre de femmes se retrouvent donc captives d'un double rôle auquel cette nouvelle forme urbaine, les banlieues en l'occurrence, est bien mal adaptée, et peu à peu les conflits inhérents à ce double rôle viennent nourrir une nouvelle crise puis une relance du mouvement des femmes. La boucle est alors bouclée, les solutions finissant par créer plus de problèmes qu'elles n'en ont résolus.

Cette vision pessimiste du développement historique n'est pas sans rappeler les analyses globalisantes de l'économie politique radicale des années 70 et leurs généralisations excessives (notamment dans l'étude des banlieues). Mais le mérite de Suzanne Mackenzie est plutôt de proposer de l'urbanisation une vision cohérente effectivement centrée sur la question des femmes. Avec l'article de Damaris Rose et de Paul Villeneuve, l'analyse se fait beaucoup plus précise et fait travailler des données statistiques de manière nuancée. Les auteurs y discutent du redéploiement économique et plus particulièrement de la restructuration de la maind'oeuvre qui ont profondément transformé le paysage métropolitain dans les années 70. Cette analyse, au départ globale, présente 
l'intérêt d'être menée à l'échelle fine des quartiers et d'être ainsi en mesure d'interroger le bien-fondé de certaines hypothèses couramment admises. Après un tour rapide de la littérature sur les phénomènes de bipolarisation et de féminisation de la main-d'oeuvre, les auteurs dressent un portrait détaillé de la spatialisation de ces phénomènes à Montréal, révélant les contrastes d'un quartier à l'autre. Dans l'analyse des facteurs de leur transformation, l'étude des contours et des effets de la gentrification devient stratégique. Rose et Villeneuve mettent à cet égard en évidence les effets de la présence de femmes professionnelles aux revenus modestes, d'une part, et de la présence d'enfants dans les ménages «gentrificateurs», d'autre part, sur le portrait socio-politique des quartiers et sur les services que l'on y retrouve.

Les propos présentés par Jeanne M. Wolfe et Grace Strachan s'inscrivent jusqu'à un certain point en continuité avec les conclusions précédentes car il y est question des contributions d'organisations féminines et de certaines de leurs illustres fondatrices aux mouvements de réforme urbaine qui ont animé la scène montréalaise au tournant du siècle. De l'Association pour les parcs et les terrains de jeu (Montreal Parks and Playgrounds Association) à la vie de cette fort progressiste lady qu'était Grace Julia Drummond, ce que cette étude historique révèle, c'est, une fois de plus, l'étroitesse des rapports entre la question urbaine et la question des femmes.

Avec le texte de William Michaelson, nous revenons au thème du double rôle des femmes, cette fois sous l'angle à la fois des horaires et de la logistique de la vie quotidienne. Et c'est hélas sans surprise que nous constatons que même les femmes salariées à plein temps consacrent encore quotidiennement près de trois fois plus de temps que leur conjoint au travail ménager et au soin des enfants. Des différences importantes apparaissent aussi dans l'utilisation des modes de transport. Les femmes utilisent davantage les transports en commun que leurs compagnons et près de $40 \%$ des femmes interrogées dans l'enquête de Toronto ne possèdent pas de permis de conduire. Ces observations, et bien d'autres, conduisent ensuite l'auteur à s'interroger sur les programmes et politiques pu- 
blics susceptibles de mieux adapter l'organisation urbaine aux exigences de la tâche des mères salariées.

Toujours dans le domaine des solutions innovatrices, Gerda Wekerle fait un bilan des expériences d'habitations coopératives gérées par des femmes dans le domaine de l'habitation, à travers le Canada. Au terme de ce bilan, elle constate que l'innovation se situe non au niveau du design mais au niveau des pratiques sociales. Cette conclusion viendrait-elle anéantir les espoirs des professionnelles de l'aménagement de voir naître un véritable design féministe? En un sens, l'article de Fran Klodowsky et Aron Spector ne contredit pas cette conclusion dans la mesure où leur évaluation des critères susceptibles d'apprécier la pertinence des conditions de logements données pour les familles monoparentales, fait ressortir en fin de compte la primauté des problèmes de revenus et de ressources auxquels sont confrontées ces familles particulières.

Le dernier article, mais non le moindre, démontre l'utilité, dans un contexte de planification, d'une compréhension exacte non seulement de la situation et des pratiques des femmes mais aussi de leurs perceptions de l'environnement et des représentations qu'elles se font de leurs propres activités. Denise Piché a choisi avec perspicacité d'explorer le domaine du loisir comme manière privilégiée de saisir la culture des femmes et de leur condition d'inégalité. Ces entrevues auprès de femmes et d'adolescentes devraient faire réfléchir plus d'un aménagiste!

Ce survol de l'état de la recherche canadienne sur les relations entre les rapports sociaux de sexe et les structures urbaines, d'abord présenté à l'occasion d'une session spéciale organisée en février 1985 par l'Institut d'études urbaines de Winnipeg dans le cadre d'un colloque sur les questions urbaines, donne une bonne idée de ce champ de recherche encore relativement récent et ténu, dont Andrew et Milroy fournissent une bibliographie annotée en fin de volume. Ce champ est certes prometteur de nouvelles lectures de la ville et de ses problèmes mais de là à reconnaître la spécificité et la nouveauté des méthodologies et outils d'analyse qui appartiendraient en propre aux études féministes, il y a une distance que le lecteur hésite à franchir, à la lecture de l'ouvrage et 
plus particulièrement du dernier chapitre que les éditrices consacrent à cette question. À moins d'être convaincu d'avance. Et dans ce champ bien précis, qui ne l'est déjà?

Annick Germain INRS-Urbanisation 